\section{P27 CHANGES IN COGNITIVE FUNCTION WITH THE MENOPAUSAL TRANSITION: A LONGITUDINAL STUDY IN ALSPAC WOMEN}

1,2 $\mathrm{F} \mathrm{Kilpi}^{*},{ }^{1,2} \mathrm{ALG}$ Soares, ${ }^{1,2,3} \mathrm{~A}$ Fraser, ${ }^{2} \mathrm{SJ}$ Fallon, ${ }^{4} \mathrm{~S}$ Nelson, ${ }^{5} \mathrm{~N}$ Sattar, ${ }^{1,2} \mathrm{~K}$ Tilling,

1,2,3DA Lawlor. 'MRC Integrative Epidemiology Unit, University of Bristol, Bristol, UK; ${ }^{2}$ Population Health Sciences, Bristol Medical School, Bristol, UK; ${ }^{3}$ Bristol NIHR Biomedical Research Centre, Bristol, UK; ${ }^{4}$ School of Medicine, Dentistry and Nursing, University of Glasgow, Glasgow, UK; ${ }^{5}$ Institute of Cardiovascular and Medical Sciences, University of Glasgow, Glasgow, UK

\subsection{6/jech-2019-SSMabstracts. 178}

Background Prior research has suggested that transient changes or an accelerated decline in cognitive function occur in women going through the menopause transition. The current evidence remains unclear as to whether these changes occur over and above those of general ageing and the extent to which they are influenced by learning from repeating the cognitive tests. The aims of this study were to (i) assess change in cognitive function by increasing reproductive age in women in mid-life, and (ii) examine the association of change in reproductive hormone levels with change in cognitive function in these women.

Methods The data were obtained from the follow-up of mothers of the Avon Longitudinal Study of Parents and Children (ALSPAC) cohort, UK. There were up to three repeat measurements of six cognitive function tests in 2,411 women (at mean ages 51, 52 and 53). The six tests covered different domains of cognitive function: processing speed, working memory, verbal episodic memory, verbal intelligence and verbal fluency. We analysed how cognitive function in different domains changed with years since final menstrual period (reproductive age) while controlling for chronological age, learning effects and socioeconomic factors using multilevel regression models. We also assessed the association of changes in reproductive hormones (luteinizing, follicle-stimulating and anti-Mullerian hormones) with changes in cognitive test performance.

Results With one exception there was little evidence of any of the cognitive function domains changing in relation to reproductive age, but processing speed showed a decline by -0.21 (95\% CI -0.34; -0.08) standard deviations (SD) per 10 years since final menstrual period. Change in reproductive hormones were not robustly associated with processing speed and other measures of cognitive function, with the exception of immediate verbal episodic memory, with both increasing follicle-stimulating and luteinizing hormone levels being associated with a decline in this domain $(-0.06$ (95\% CI $-0.09 ;-0.02)$ and $-0.06 \quad(95 \%$ CI $-0.10 ;-0.02) \quad$ SD change, respectively).

Discussion We found little evidence for reproductive age or change in reproductive hormones having an important effect on women's cognitive function in mid-life. Of the 24 associations estimated, there was evidence of decline in two of the six cognitive domains, but these were not consistent between reproductive age and reproductive hormones.

\section{P28 MENTAL HEALTH INEQUALITIES AMONG MEN WITH DISABILITY: AN INTERSECTIONAL APPROACH}

${ }^{1} \mathrm{TL}$ King*, ${ }^{1} \mathrm{M}$ Shields, ${ }^{2} \mathrm{~T}$ Shakespeare, ${ }^{1} \mathrm{~A}$ Milner, ${ }^{1} \mathrm{~A}$ Kavanagh. ${ }^{1}$ Disability and Health Unit, Centre for Health Equity, Melbourne School of Population and Global Health, The University of Melbourne, Carlton, Australia; ${ }^{2}$ International Centre for Evidence in Disability, London School of Hygiene and Tropical Medicine, London, UK

\subsection{6/jech-2019-SSMabstracts. 179}

Background Disability is a key social identity or social category that is associated with significant social disadvantage. For men, having a disability can be discordant with their masculine identity. Self-reliance is one component of masculinity that is known to be important to men with disabilities, however it is also known to be associated with adverse mental health outcomes in the broader adult male population. Intersectionality approaches offer a means of examining the way that the effect of self-reliance on mental health might vary between those with and without a disability.

Methods We drew on a sample of 12,052 men aged 18-55 years from the Ten-to-Men study, a longitudinal cohort study of Australian men and boys. We used survey-weighted adjusted Poisson regression to examine associations between disability and depressive symptoms, adjusting for age, country of birth, indigenous status, education and area disadvantage. We applied an intersectional approach by using effect measure modification (EMM) to examine how conformity to the masculine norm of self-reliance modifies the relationship between disability and depressive symptoms, calculated on both the multiplicative and additive scales.

Results Results showed that men with disabilities who reported higher conformity to self-reliance norms had worse mental health than non-disabled men with low conformity to self-reliance, as measured in terms of depressive symptoms (PRR: 9.40, 95\%CI 7.88, 11.22). We found evidence of positive EMM of depressive symptoms by conformity to self-reliance on the additive scale (RERI: $2.84,95 \%$ CI 1.26, 4.42).

Conclusion These results highlight the mental health differences between men with and without disabilities, and provide evidence that high conformity to self-reliance norms exerts a particularly damaging effect on the mental health of men with disabilities. Given that men with disabilities are more likely to rely on help and support from others, these results have important implications for the delivery of services to men with disabilities. The results suggest a role of practitioners and advocates in encouraging men with disabilities to accept support when needed, and also advocate for them to be able to access technologies and other supports that enable them to be more self-reliant. 